\title{
Ectomycorrhizal fungal diversity and saprotrophic fungal diversity are linked to different tree community attributes in a field-based tree experiment
}

\author{
NHU H. NGUYEN, * LAURA J. WILLIAMS, † JOHN B. VINCENT, * ARTUR STEFANSKI, \\ JEANNINE CAVENDER-BARES, $\uparrow$ CHRISTIAN MESSIER, $\S$ ALAIN PAQUETTE, $\S$ \\ DOMINIQUE GRAVEL, PETER B. REICH $\$$ and PETER G. KENNEDY*† \\ *Department of Plant Biology, University of Minnesota, St. Paul, MN, USA, †Department of Ecology, Evolution and Behavior, \\ University of Minnesota, St. Paul, MN, USA, $\$$ Department of Forest Resources, University of Minnesota, St. Paul, MN, USA, \\ $\S$ Department of Biological Sciences, University of Quebec, Montreal, QC, Canada, $\mid$ Department of Biology, University of \\ Sherbrooke, Sherbrooke, QC, Canada
}

\begin{abstract}
Exploring the link between above- and belowground biodiversity has been a major theme of recent ecological research, due in large part to the increasingly well-recognized role that soil microorganisms play in driving plant community processes. In this study, we utilized a field-based tree experiment in Minnesota, USA, to assess the effect of changes in plant species richness and phylogenetic diversity on the richness and composition of both ectomycorrhizal and saprotrophic fungal communities. We found that ectomycorrhizal fungal species richness was significantly positively influenced by increasing plant phylogenetic diversity, while saprotrophic fungal species richness was significantly affected by plant leaf nitrogen content, specific root length and standing biomass. The increasing ectomycorrhizal fungal richness associated with increasing plant phylogenetic diversity was driven by the combined presence of ectomycorrhizal fungal specialists in plots with both gymnosperm and angiosperm hosts. Although the species composition of both the ectomycorrhizal and saprotrophic fungal communities changed significantly in response to changes in plant species composition, the effect was much greater for ectomycorrhizal fungi. In addition, ectomycorrhizal but not saprotrophic fungal species composition was significantly influenced by both plant phylum (angiosperm, gymnosperm, both) and origin (Europe, America, both). The phylum effect was caused by differences in ectomycorrhizal fungal community composition, while the origin effect was attributable to differences in community heterogeneity. Taken together, this study emphasizes that plant-associated effects on soil fungal communities are largely guild-specific and provides a mechanistic basis for the positive link between plant phylogenetic diversity and ectomycorrhizal fungal richness.
\end{abstract}

Keywords: fungal guild, geographic origin, host specificity, microbial richness, plant phylogenetic diversity

Received 15 December 2015; revision received 23 May 2016; accepted 26 May 2016

\section{Introduction}

Understanding the link between above- and belowground biodiversity has been a major theme of recent

Correspondence: Peter G. Kennedy, Fax: 612625 1738; E-mail: kennedyp@umn.edu ecological research, due in large part to the increasingly well-recognized role that soil microorganisms play in driving plant community productivity (Van der Heijden et al. 2008), vegetational succession dynamics (Van der Putten et al. 1993; Nara \& Hogetsu 2004) and ecosystem functioning (Bardgett \& Van der Putten 2014). It is now widely accepted that strong feedbacks exist between 
plant and soil microbial communities, although the direction and intensity of those feedbacks are spatially (De Deyn \& Van der Putten 2005), temporally (Eisenhauer et al. 2011) and environmentally context dependent (Smith \& Reynolds 2015). Along with better understanding the basic functioning of ecosystems, determining the extent to which above- and belowground communities are coupled also has significant conservation implications (Wardle 2006; Wagg et al. 2014).

One of the most fundamental aspects of the link between plant and soil microbial communities is the extent to which above- and belowground species richness are interdependent. Many of the theoretical treatments of this topic suggest that the relationship between plant and soil microbial richness should be positive due to greater heterogeneity in resources and environmental conditions as plant richness increases (Hooper et al. 2005; Wardle 2006). Despite a well-established conceptual foundation, and evidence for a positive relationship between plant richness and aboveground food web richness (Haddad et al. 2009), the effect of plant richness on soil microbial richness remains equivocal. A number of field-based surveys indicate that bacterial species richness is actually poorly predicted by plant species richness (Fierer \& Jackson 2006; Bryant et al. 2008; Prober et al. 2014), with environmental variables such as soil $\mathrm{pH}$ being more significant ecological drivers (Lauber et al. 2009). For soil fungi, which may be more strongly influenced by plant effects on soil due to their growth at larger spatial scales (Urbanová et al. 2015), the evidence is currently mixed. Tedersoo et al. (2014a) found that abiotic factors, particularly mean annual precipitation, were significantly more important than plant species richness in determining global hotspots of soil fungal richness. Similarly, Prober et al. (2014) found no consistent relationship between plant and soil fungal richness at 25 grassland sites located on four continents. In contrast, Gao et al. (2013) found that ectomycorrhizal fungal richness, a dominant fungal guild in many forests globally, increased significantly with increasing plant species richness in both temperate and tropical forests. At local spatial scales, positive correlations between plant and soil fungal richness have been observed in both forest (Peay et al. 2013; Tedersoo et al. 2015) and grassland ecosystems (Pellisier et al. 2014).

An inherent challenge of field surveys of plant-soil microbial richness relationships is the correlation of species richness with other environmental variables. For example, Landis et al. (2004) found that arbuscular mycorrhizal fungal richness was positively associated with plant species richness, but both also increased with greater nitrogen availability and changes in soil texture.
Disentangling this relationship can often be achieved with experimental plantings of plant species, but those can also have shortcomings, particularly if monocultures are not present to account for sampling effects (Huston 1997; Tedersoo et al. 2014b). In the field-based studies with the proper set of experimental treatments, there has been general support for a strong relationship between plant and soil fungal communities. Both Burrows \& Pfleger (2002) and Hiiesalu et al. (2014) found that increasing plant species richness resulted in a significant increase in the richness of arbuscular mycorrhizal fungal communities. Working in the same study system, Antoninka et al. (2011) found arbuscular mycorrhizal fungal richness was higher in mono- and polyculture plots, but LeBlanc et al. (2015) recently found the reversed pattern for the bulk soil fungal community. Glasshouse studies explicitly manipulating plant and soil fungal community richness have also typically found a strong relationship (Van der Heijden et al. 1998, Vogelsang et al. 2006). Despite this growing consensus, nearly all of the experimental studies examining this relationship have focused on herbaceous plant communities, particularly grasses. For tree-based ecosystems, Tedersoo et al. (2015) recently documented a strong relationship between tree and ectomycorrhizal fungal richness at one experimental site (Finland) but no relationship at another (Estonia). Given the divergence in response between sites, determining the nature of this relationship in other experimental forest settings is important for making broader ecological predictions.

Despite the greater study of the links between plant and microbial community richness and composition, the mechanisms underlying these trends remain largely unresolved. Some research suggests that the positive relationships between plant and soil fungal community richness are not driven by the number of plant species, but rather by the increased productivity in plots with higher plant species richness (Waldrop et al. 2006). A recent study in which plant biomass (a traditional measure of productivity) was carefully measured belowground, however, found that increases in belowground plant biomass (and species richness) were actually negatively correlated with arbuscular mycorrhizal fungal community richness (Hiiesalu et al. 2014). Those results suggest the effect of plant productivity on fungal richness may be system- and measurement-type dependent. An intuitive but less well-discussed alternative factor driving positive relationships between plant and soil fungal community richness is differential host specificity, which would result in higher fungal richness in plots with more plant species. Support for the role of host specificity in increasing fungal richness has been shown in observational ectomycorrhizal fungal community studies (Kernaghan et al. 2003, Ishida et al. 2007; 
Tedersoo et al. 2013; Gao et al. 2013), as well as experimental studies of fungal pathogens (Rottstock et al. 2014), although the absence of monoculture plots have often confounded this interpretation (Tedersoo et al. 2014a; but see Gao et al. 2014). Work in plant-fungal pathogen systems also indicates that specificity-richness relationships may be strongly mediated by host phylogenetic relationships (Gilbert \& Webb 2007), which would suggest that plots containing more phylogenetically distant plant species should have higher fungal richness than those with the same number of more closely related plant hosts. Indirect support for this effect comes from the recent study of global fungal richness patterns, where ectomycorrhizal fungal richness peaked in temperate forests, which contained higher host phylogenetic diversity than ectomycorrhizal-dominated tropical forests (Tedersoo et al. 2014b).

The recent surge in research on links between aboveand belowground species communities has been facilitated by the application of molecular techniques, which have allowed ecologists to more accurately quantify microbial community richness and composition (Peay et al. 2008; Caporaso et al. 2011). Importantly, current methods allow researchers to quantify entire microbial communities rather just individual functional guilds (Nguyen et al. 2016). Differences among guilds in their response to plant richness might be expected, particularly between biotrophic guilds that directly interact with plants and saprotrophic guilds that interact with plants indirectly (Urbanová et al. 2015). Support for a guild-level difference was shown by Peay et al. (2013) and at the Finnish site in Tedersoo et al. (2015), where the richness of symbiotic soil fungal guilds was positively associated with higher plant richness, but the richness of saprotrophic fungi was not. However, both Prober et al. (2014) and Pellisier et al. (2014) found contrasting results, with the richness of both mutualistic and total soil fungal communities not being significantly affected by plant richness.

In this study, we utilized a field-based tree experiment to test the link between plant and soil fungal community richness and composition. The design of the experimental site we sampled, which includes both mono- and different kinds of mixed-species plots, allowed us to account directly for sampling effects, while testing the effect of plant species richness, phylogenetic diversity and range of additional biotic and abiotic parameters on soil fungal richness and composition. In addition, the experiment includes pairings of tree species from multiple tree genera, in which one tree species was native to North America, while the other was native to Europe. This phylogenetically controlled design also allowed for an explicit testing of the effect of plant origin on soil fungal community richness and composition. Based on previous observational studies, we hypothesized that plant and soil fungal community richness would be positively correlated, with the greatest effects being apparent in mixed-host species plots with higher plant phylogenetic diversity. If fungal host specificity was the important mechanism driving this relationship, we also expected to see many fungal species present only in the mono- and mixed-species plots containing their specific host species. With regard to the effects of plant origin, we anticipated higher fungal richness in the plots with North American hosts compared with those from Europe, due to a greater likelihood of compatible symbionts being present. Finally, similar to fungal species richness, we expected that patterns of fungal community composition would also be significantly influenced by both plant phylogenetic diversity and plant origin. Given the potential for differential responses to plant species richness and composition among fungal guilds, we examined the aforementioned predictions for both ectomycorrhizal and saprotrophic fungal communities.

\section{Methods}

\section{Experiment design and environmental sampling}

This study conducted in the high-density tree-diversity experiment established in Cloquet, Minnesota, USA, as part of the International Diversity Experiment Network with Trees (IDENT, Tobner et al. 2014). In the spring of 2010, 9408 tree seedlings were planted at the Cloquet IDENT site (Fig. S1, Supporting information). Six common North American temperate-boreal tree species were planted (Acer saccharum, Betula papyrifera, Larix laricina, Picea glauca, Pinus strobus and Quercus rubra) along with a congener of each species originating from Europe (A. platanoides, B. pendula, L. decidua, Picea abies, Pinus sylvestris and $Q$. robur). Trees were planted in a grid pattern with $0.4 \mathrm{~m}$ spacing among seedlings to form plots $2.8 \times 2.8 \mathrm{~m}$ in size containing 49 trees. Plots were spaced one $\mathrm{m}$ apart. Here, we focused on a subset of the plots in the experiment: those plots where each species was growing in monoculture and four six-species mixtures. The latter contained either (i) six North American species, (ii) six European species, (iii) six gymnosperms or (iv) six angiosperms, respectively. Each of these plots was replicated four times within the experiment in a randomized block design.

We characterized the tree communities and environmental conditions of each plot in several ways, using the core area containing 25 trees (to minimize edge effects). We calculated plot plant species richness by counting the number of plant species present. We used the phylogenetic species variability metric (PSV; 
Helmus et al. 2007) to quantify plot plant phylogenetic diversity (calculated in $\mathrm{R}$ using the PICANTE package). Unlike other phylogenetic diversity metrics such as Faith's PD, PSV is independent of species richness (Pearse et al. 2014). To estimate standing biomass in the plots, we measured the stem basal diameter $(D)$ and stem height $(H)$ for each tree at the end of the 2013 growing season. These measurements along with wood density (WD) values obtained from literature (Chave et al. 2009) were used to calculate standing biomass ( $D^{2}$ $\times H \times$ WD). To assess specific root length in the plots, we calculated a community-weighted mean using in situ measurements. In June 2014, we sampled trees in each monoculture plot growing in the outside row. For each tree, we analysed scanned images of three to five subsamples of first- to third-order roots (following Pregitzer et al. 2002) using WinRhizo (Regent Instruments Inc., Quebec, Canada). Specific root lengths for each species were calculated as the average of measurements taken on six trees and the community-weighted specific root length was calculated by weighting species' mean values by the proportion of trees on a plot that belonged to each species.

We assessed plot leaf nitrogen concentration using the same community-weighted approach as for specific root length. For this measure, one fully expanded, sunexposed leaf (or five fascicles for Pinus and approx. 20 needles for Picea and Larix spp.) was collected in June 2013 from each of five trees chosen at random in each monoculture plot and pooled to give one sample per species per block. Each sample was dried at $65^{\circ} \mathrm{C}$, finely ground and analysed for total nitrogen and carbon at the University of Nebraska, Lincoln, using a Costech ECS 4010 element analyser. We estimated plot leaf area index (LAI) during peak biomass (the last two weeks of August 2013) using a line intercept method. Fifteen vertical lines were located randomly on each plot. For each line, the species identity was recorded for each leaf or gymnosperm branchlet intercepted, and the angle of each leaf or branchlet intercepted was measured to the nearest 15 degrees. A subsample of gymnosperm branchlets was destructively sampled to estimate the ratio of interceptable to total leaf area (Thomas \& Winner 2000). For all of the aforementioned measurements, we calculated plot values in two ways, once including all of the tree species on the plot and again excluding the two species that were not ectomycorrhizal hosts (i.e. A. saccharum and A. platanoides).

To assess the physical environment, we estimated plot surface soil moisture (\% volumetric water content) by averaging six measurements from the start of June and then two-week intervals from the start of July to mid-August 2013. Measurements were taken to $12 \mathrm{~cm}$ depth at four locations per plot using time domain reflectometry with a FieldScout TDR 300 (standard soil type; Spectrum Technologies Inc., Illinois, USA). We determined plot soil $\mathrm{pH}$ from the soil samples collected for fungal molecular analyses using a water-based laboratory method (Soil Survey Staff 2004). We estimated the standing pools of two forms of inorganic, plantavailable nitrogen (nitrate and ammonium) at the end of June 2013. Two soil cores $(2.54 \mathrm{~cm}$ diameter and $15 \mathrm{~cm}$ deep) were collected from random locations on each plot using a PVC corer and pooled to give one sample per plot. Samples were collected, sieved, homogenized and extracted with $2 \mathrm{M} \mathrm{KCl}$ within $48 \mathrm{~h}$. Extracts were analysed for nitrate and ammonium using colorimetric assays (Hood-Nowotny et al. 2010).

\section{Fungal sampling and molecular identification}

On 28 October 2013, we collected soil cores from each of the four replicate plots of the 16 tree species treatments $(n=64)$. We first removed the surface litter layer and then took four individual $2.5 \times 10 \mathrm{~cm}$ deep cores located $0.8 \mathrm{~m}$ in from each plot edge (all cores were taken with surface-sterilized PVC cores to prevent any cross contamination). The four cores were combined into a single plastic bag and immediately stored on ice. We returned the soil samples to the laboratory the same day and processed them for molecular analyses within $48 \mathrm{~h}$ of field collection.

Soils were homogenized by shaking and then sieved through a 2-mm surface-sterilized mesh (to remove roots and other plant material). DNA was extracted from $10 \mathrm{~g}$ of soil per sample using the PowerMax Soil DNA Isolation Kit (MO Bio, Carlsbad, CA, USA) according to the manufacturer's instructions. The ITS1 rDNA subunit was amplified using a barcoded fungalspecific ITS1F-ITS2 primer set (Smith \& Peay 2014). For each sample, we ran three individual PCRs at different annealing temperatures $\left(55,56.5,58^{\circ} \mathrm{C}\right)$, which were pooled to minimize the possibility of annealing temperature biases. Along with the experimental samples, we included a series of field, laboratory extraction and PCR-negative controls as well as a mock community as a positive control (Nguyen et al. 2015). Amplified products were magnetically cleaned using the Agencourt AMPure XP Kit (Beckman Coulter, Brea, CA, USA) and quantified using the Qubit dsDNA HS Fluorometer (Life Technologies, Carlsbad, CA, USA) according to manufacturer's instructions. The final library was sequenced at the University of Minnesota Genomics Center using the 250-bp paired-end MiSeq Illumina platform. Raw sequence data and associated metadata were deposited in MG-RAST under project number 13302. 
Raw sequences were demultiplexed, quality-filtered using Phred $=20$ and culled of sequences $<125 \mathrm{bp}$, with any ambiguous bases, or a homopolymer run of $>8 \mathrm{bp}$ using QIIME v 1.8 (Caporaso et al. 2011) and мотнUR v 1.33.3 (Schloss et al. 2009). In this particular run, the reverse reads had poor quality and pairing them with forward reads notably reduced the final number of sequences (Nguyen et al. 2015). Therefore, we chose to use only the forward reads for all of the final analyses. Following the guidelines discussed in Nguyen et al. (2015), we employed a multistep operational taxonomic unit (OTU) picking strategy in QIIME by first clustering with reference USEARCH (including reference and de novo chimera checking) at a $95 \%$ sequence similarity followed by denovo UCLUST at $95 \%$ sequence similarity. We found that these criteria best recovered the mock community and therefore applied them to the rest of the samples. The UNITE v6 database (Kõljalg et al. 2013; http://unite.ut.ee/repository.php) was used in chimera checking and OTU clustering. OTUs were identified using NCBI BLAST+ v2.2.29 (Altschul et al. 1990) and a custom database with sequences from the UNITE v6 and vouchered mushrooms collected from the field site. As sequences with a low match length/query length ratio were typically nonfungal or contained a very short fungal segment, we kept only sequences had a length/qlen $\geq 0.845$ from BLAST. OTUs that occurred in the negative controls were removed by subtracting the number of sequences of each OTU present in the negative controls from the sequence abundance of that OTU in the experimental samples. To account for differences in sequence reads among samples, all samples were rarefied to 30300 sequences, which represented the lowest total in one of our 64 samples. In preliminary data analyses, we compared this approach to that used by Tedersoo et al. (2015), which was based on residual richness after accounting for sequence reads and OTU richness. The results were similar, suggesting that our rarefaction approach did not generate any notable bias (McMurdie \& Holmes 2014). The final raw community matrices, including taxonomic identification for each OTU (hereafter referred to as species), are provided in Tables S1 (ectomycorrhizal fungi) and S2 (saprotrophic fungi) (Supporting information).

\section{Statistical analyses}

While the use of high-throughput sequencing has become a standard way to study soil fungal communities, there is growing recognition that multiple quality control steps are needed to ensure ecological accurate analyses (Lindahl et al. 2013; Nguyen et al. 2015; Song et al. 2015). To address the issue of false positives in our data (i.e. sequences present in samples due to spurious biological sources and/or sequencing artefacts such as tag switching (Carlsen et al. 2012)), we began by examining the single-species Acer plots, which do not host ectomycorrhizal fungi. Over $70 \%(842 / 1176)$ of the sample-by-species combinations had no ectomycorrhizal fungal sequences, but 95 of the 147 ectomycorrhizal species were detected at least once in the Aceronly plots. Despite this relatively high frequency, the number of ectomycorrhizal fungal sequences per species was 1.5 orders of magnitude lower than plots containing ectomycorrhizal host trees (Acer-only zeroexcluded mean $=23$ reads, ectomycorrhizal host zeroexcluded mean $=409$ reads). Given the differences in sequence abundance, we believe the ectomycorrhizal fungal sequences present in the Acer-only plots were not likely due to living mycelia, but rather the product of ectomycorrhizal fungal spores and/or tag switching (in a separate study looking at mushrooms at the Cloquet IDENT site, we noticed almost no fruiting between plots, suggesting root growth in from adjacent plots at the time of sampling was not a significant issue). Because ectomycorrhizal spores/tag switching would also be present in plots with ectomycorrhizal host vegetation, determining the number of sequence reads that indicate an ectomycorrhizal fungal species is actively growing in the plot rather than simply present as spores or via tag switching is essential for accurately assessing plant-associated patterns of fungal richness (and for saprotrophic fungi as well). Specifically, the inclusion of these false positives would lead to overestimates of plant association for fungal species (i.e. an absence of specificity). As our DNA-based approach did not allow us to molecularly parse these two source pools (see Van der Linde \& Haller (2013) for an RNAbased alternative regarding spores), we employed a simulation approach instead.

We examined the ectomycorrhizal sequence abundance data from the 40 single-species ectomycorrhizal host plots. Looking at the sequence abundances of known ectomycorrhizal fungal specialists in our data set [i.e. species only associated with certain host genera (Molina et al. 1992)], values above 21 sequences per sample appeared to clearly represent active growth rather than presence as spores or switched tags (i.e. in the majority of the samples where specialist taxa were present with their specific hosts, the number of sequence reads was at or well above 21 sequences). In a small number of cases, however, values below 21 also looked biologically real (i.e. a specialist was present in only the plots it should have been, but with an abundance below 21). As such, we simulated the number of plots each ectomycorrhizal fungal species was present in using a rolling cut-off that increased from 1 to 21 in two sequence read increments (Fig. S2, Supporting 
information, $\mathrm{R}$ code available upon request). Comparing the distributions of the 10 increment steps, we found that when samples with one and three sequence reads were included, the number of plots that ectomycorrhizal fungal species were present in was significantly different from the majority of the other cut-offs. As five sequences was the lowest cut-off that was not significantly different from 21 sequences in our simulation, we decided to use that value as a conservative threshold for taxon inclusion in the species richness analyses (this value also matches the recommendations of Lindahl et al. (2013)). Because saprotrophic fungi have similar spore dispersal strategies (i.e. many produce mushrooms like ectomycorrhizal fungi) and would likely have the same issues with tag switching, we applied the same five sequence read cut-off to the richness analyses for that data set as well.

To assess the effects of different continuous biotic and abiotic factors on ectomycorrhizal and saprotrophic fungal species richness, we used a linear modelling approach. Due to shortcomings-associated stepwise model selection (Whittingham et al. 2006), we used information theory model selection in the 'MUMIN' package in $\mathrm{R}$ ( $\mathrm{R}$ Core Team 2014). The models assessing ectomycorrhizal and saprotrophic fungal richness were identical in terms of type and number of predictor variables, but differed in replication due to the lack of ectomycorrhizal associations with Acer trees. As a result, we did not include the Acer-only plots in the ectomycorrhizal model and when counting plant species richness, for example, the number of species was four in the ectomycorrhizal fungal model (excluding both Acer species) and six in the saprotrophic fungal model (including both Acer species) in the six angiosperm treatment plots. One Quercus rubra plot in which soil moisture was a significant outlier from all other plots was also excluded.

For both the ectomycorrhizal and saprotrophic richness models, we included nine predictor variables: plant species richness, plant phylogenetic diversity, plant standing biomass, plant leaf area index (LAI), plant specific root length, plant leaf nitrogen, soil water content, soil nitrogen and soil $\mathrm{pH}$ (see Table 1 and above for calculations/units). Due to differences in the ranges of each variable, all were first standardized (using the 'scale' function). We then used the $1 \mathrm{~m}$ function to fit a basic linear model for each response variable (i.e. ectomycorrhizal or saprotrophic richness) and determined that there were no significant issues with multicollinearity among variables using the 'FMSB' package (all VIC scores <2.4). Using the 'DREDGE' function in MUMIN, we then generated a full set of models for each response variable (ectomycorrhizal or saprotrophic richness) that varied in inclusion of the nine predictor variables (Tables S3 and S4, Supporting information). Those models were then ranked and all those within four $\mathrm{AIC}_{\mathrm{c}}$ units of the best fitting model (i.e. with the lowest $\mathrm{AIC}_{\mathrm{C}}$ score) were further compared using model averaging. (We also assessed those within a 95\% confidence interval of the best fitting model and obtained the same results (Tables S5 and S6, Supporting information) The beta coefficients, standard errors, confidence intervals, significance and relative importance values reported in Table 1 are based on the top 38 and 12 models for ectomycorrhizal and saprotrophic richness, respectively (additional details on each of the models with the lowest AICc score for each fungal guild are presented in Tables S7 and S8, Supporting information). We also explored the effect of adding interaction terms to each model, but none significantly improved the global $\mathrm{AIC}_{\mathrm{c}}$ scores, so were not included.

To assess the effect of the three categorical variables on ectomycorrhizal and saprotrophic fungal richness, we also examined sampling block (A, B, C, \& D), host origin (Europe \& America), and host phylum (gymnosperm, angiosperm, both). We used a three-way ANOVA for each fungal group, with no interaction terms (due to loss of degrees of freedom). For consistency between the continuous and categorical variable analyses, the one Quercus rubra plot in which soil moisture was a significant outlier from all other plots remained excluded. Both ANOVAS were confirmed to meet model assumptions by checking residual and Q$Q$ plots. Differences among of treatment means for significant variables were determined using Tukey HSD tests.

We also assessed the effects of plant species composition, plant phylum and plant origin on changes in fungal species composition for both guilds. We first compared the relationship between plant and fungal species composition for ectomycorrhizal and saprotrophic fungi using Mantel tests. These analyses were run using both presence-absence and abundance-based data. As the results were functionally equivalent (data not shown), only the abundance-based data are presented. Prior to the Mantel tests, the fungal data were Hellinger-transformed and dissimilarities were calculated using the Bray-Curtis metric. We additionally assessed the effect of changes in plant species composition using the Raup-Crick metric (Chase et al. 2011), which better accounts for the changes in alpha diversity across our experimental design (i.e. 1 vs. 4, 5, or 6 plant species in the ectomycorrhizal and saprotrophic fungal analyses). The effect of plant phylum and plant origin on both ectomycorrhizal fungal and saprotrophic fungal species composition were compared using PERMANOVA with complete randomization. To determine whether significant differences were based on centroid location 
Table 1 Effects of nine continuous abiotic and biotic variables on ECM and saprotrophic fungal richness in the Cloquet IDENT experiment

\begin{tabular}{|c|c|c|c|c|}
\hline Variable & Parameters $(n=55)$ & ECM fungal richness & Parameters $(n=63)$ & SAP fungal richness \\
\hline Plant species richness & $\begin{array}{l}\text { Range }=1-6 \\
\#=4 \text { different values }\end{array}$ & $\begin{array}{l}\mathrm{MAE}=0.45 \pm 0.93 \\
\mathrm{CI}=-1.39,2.30 \\
P=0.629 \mathrm{RVI}=0.11\end{array}$ & $\begin{array}{l}\text { Range }=1-6 \\
\#=2 \text { different values }\end{array}$ & $\begin{array}{l}\mathrm{MAE}=1.36 \pm 1.28 \\
\mathrm{CI}=-1.20,3.92 \\
P=0.296 \\
\mathrm{RVI}=0.36\end{array}$ \\
\hline Plant phylogenetic diversity & $\begin{array}{l}\text { Range }=0-0.65 \\
\#=4 \text { different values }\end{array}$ & $\begin{array}{l}\mathrm{MAE}=1.44 \pm 0.62 \\
\mathrm{CI}=0.19,2.68 \\
\boldsymbol{P}=\mathbf{0 . 0 2 3} \\
\mathrm{RVI}=0.98\end{array}$ & $\begin{array}{l}\text { Range }=0-0.65 \\
\#=2 \text { different values }\end{array}$ & $\begin{array}{l}\mathrm{MAE}=-0.85 \pm 1.50 \\
\mathrm{CI}=-3.83,2.14 \\
P=0.579 \\
\mathrm{RVI}=0.15\end{array}$ \\
\hline Plant standing mass & $\begin{array}{l}\text { Range }=997-23913 \\
\#=55 \text { different values }\end{array}$ & $\begin{array}{l}\mathrm{MAE}=-1.13 \pm 0.73 \\
\mathrm{CI}=-2.58,0.32 \\
P=0.127 \\
\mathrm{RVI}=0.41\end{array}$ & $\begin{array}{l}\text { Range }=317-23913 \\
\#=64 \text { different values }\end{array}$ & $\begin{array}{l}\mathrm{MAE}=-3.65 \pm 1.38 \\
\mathrm{CI}=-6.41,-0.88 \\
\boldsymbol{P}=\mathbf{0 . 0 0 9} \\
\mathrm{RVI}=1.00\end{array}$ \\
\hline Plant leaf area index & $\begin{array}{l}\text { Range }=04.7-5.68 \\
\#=50 \text { different values }\end{array}$ & $\begin{array}{l}\mathrm{MAE}=1.18 \pm 0.75 \\
\mathrm{CI}=-0.32,2.69 \\
P=0.123 \\
\mathrm{RVI}=0.48\end{array}$ & $\begin{array}{l}\text { Range }=04.7-5.68 \\
\#=57 \text { different values }\end{array}$ & $\begin{array}{l}\mathrm{MAE}=-0.09 \pm 1.79 \\
\mathrm{CI}=-3.67,3.49 \\
P=0.960 \\
\mathrm{RVI}=0.11\end{array}$ \\
\hline Plant leaf nitrogen & $\begin{array}{l}\text { Range }=0.89-2.52 \\
\quad \#=24 \text { different values }\end{array}$ & $\begin{array}{l}\mathrm{MAE}=-0.51 \pm 0.79 \\
\mathrm{CI}=-2.10,1.35 \\
P=0.526 \\
\mathrm{RVI}=0.12\end{array}$ & $\begin{array}{l}\text { Range }=0.89-2.52 \\
\#=21 \text { different values }\end{array}$ & $\begin{array}{l}\text { MAE }=6.69 \pm 1.44 \\
\text { CI }=3.81,9.58 \\
P<\mathbf{0 . 0 0 1} \\
\text { RVI }=1.00\end{array}$ \\
\hline Plant specific root length & $\begin{array}{l}\text { Range }=1.01-154.33 \\
\#=24 \text { different values }\end{array}$ & $\begin{array}{l}\text { MAE }=0.99 \pm 0.65 \\
\mathrm{CI}=-0.39,2.19 \\
P=0.172 \\
\mathrm{RVI}=0.38\end{array}$ & $\begin{array}{l}\text { Range }=20.41-75.33 \\
\#=27 \text { different values }\end{array}$ & $\begin{array}{l}\text { MAE }=-5.21 \pm 1.30 \\
\mathrm{CI}=-7.77,-2.65 \\
\boldsymbol{P}<\mathbf{0 . 0 0 1} \\
\mathrm{RVI}=1.00\end{array}$ \\
\hline Soil nitrogen & $\begin{array}{l}\text { Range }=0.21-9.42 \\
\#=49 \text { different values }\end{array}$ & $\begin{array}{l}\mathrm{MAE}=0.17 \pm 0.59 \\
\mathrm{CI}=-1.02,1.35 \\
P=0.784 \\
\mathrm{RVI}=0.06\end{array}$ & $\begin{array}{l}\text { Range }=0.21-9.42 \\
\#=55 \text { different values }\end{array}$ & $\begin{array}{l}\mathrm{MAE}=-0.14 \pm 1.09 \\
\mathrm{CI}=-2.32,2.04 \\
P=0.896 \\
\mathrm{RVI}=0.11\end{array}$ \\
\hline Soil water & $\begin{array}{l}\text { Range }=8.5-17.2 \\
\#=54 \text { different values }\end{array}$ & $\begin{array}{l}\mathrm{MAE}=0.67 \pm 0.61 \\
\mathrm{CI}=-0.56,1.90 \\
P=0.284 \\
\mathrm{RVI}=0.26\end{array}$ & $\begin{array}{l}\text { Range }=8.5-22.3 \\
\#=61 \text { different values }\end{array}$ & $\begin{array}{l}\mathrm{MAE}=0.48 \pm 1.19 \\
\mathrm{CI}=-1.91,2.87 \\
P=0.692 \\
\mathrm{RVI}=0.12\end{array}$ \\
\hline Soil pH & $\begin{array}{l}\text { Range }=4.67-6.04 \\
\#=39 \text { different values }\end{array}$ & $\begin{array}{l}\text { MAE }=-1.58 \pm 2.13 \\
\mathrm{CI}=-5.85,2.69 \\
P=0.468 \\
\mathrm{RVI}=0.14\end{array}$ & $\begin{array}{l}\text { Range }=4.67-6.04 \\
\#=55 \text { different values }\end{array}$ & $\begin{array}{l}\mathrm{MAE}=-1.67 \pm 3.89 \\
\mathrm{CI}=-9.47,6.11 \\
P=0.673 \\
\mathrm{RVI}=0.11\end{array}$ \\
\hline
\end{tabular}

Variable Plot level measure

Species richness Species richness Species richness

Leaf area index

Leaf nitrogen

Specific root length

Soil nitrogen

Soil water

Soil $\mathrm{pH}$
Host (ECM) or plant (SAP) species

Helmus' phylogenetic species variability

Aboveground biomass (g) (DBH $\times$ height $\times$ wood density), October 2013

Leaf $\left(\mathrm{m}^{2}\right)$ /ground $\left(\mathrm{m}^{2}\right)$, peak biomass August 2013

Dry mass (\%), September 2013

Root length $(\mathrm{m} / \mathrm{g})$ based on single-species plots, June 2014

$[\mathrm{NH} 4+\mathrm{NO} 3] \mathrm{mg} / \mathrm{kg}$, June 2013

Average volumetric content (\%), June-August 2013

Water-based pH, October 2013

MAE, model-averaged coefficient estimate \pm unconditional standard error, $\mathrm{CI}$, confidence interval $(2.5 \%, 97.5 \%) P=$ significance $(<0.05$ in bold), RVI, relative variable importance. Because of variable standardization ahead of modeling, the model-averaged estimates are directly comparable and their magnitude represents a proxy for relative effect size. Ectomycorrhizal richness model $r^{2}=0.20$, Saprotrophic richness model $r^{2}=0.31$.

or dispersion, we used the 'BETADISPER' function. Variation in composition among plots was visualized for each guild (ectomycorrhizal vs. saprotroph) and variable (plant phylum vs. plant origin) using nonmetric multidimensional scaling (NMDS) on Bray-Curtis dissimilarity matrices. All assessments of the 
relationships between plant and fungal composition were run in the VEGAN package in $\mathrm{R}$.

\section{Results}

From the total of 3508787 sequences remaining after bioinformatics filtering and quality control, 2842254 $(81 \%)$ belonged to ectomycorrhizal fungal species and $666533(19 \%)$ belonged to saprotrophic fungal species. Across the two guilds, we detected a total of 457 fungal species in the final rarefied data set; 147 were ectomycorrhizal and 310 were saprotrophic. The average number of sequences per plot was 15107 for ectomycorrhizal and 6415 saprotrophic fungal species, respectively. Species accumulation curves showed that plots were well saturated for both guilds (Fig. S3, Supporting information), with an average richness of 18 ectomycorrhizal fungal species and 38 saprotrophic fungal species.

In the statistical models assessing the effects of continuous biotic and abiotic factors on fungal richness, the outcomes differed by guild (Table 1). Ectomycorrhizal fungal richness was significantly positively associated with host phylogenetic diversity, but not with any other factors (Fig. S4, Supporting information). Host phylogenetic diversity dominated the lowest-AIC scoring models, having a relative importance value of $93 \%$ (the next closest was host LAI at $48 \%$ ). Unlike ectomycorrhizal fungal richness, saprotrophic fungal richness was significantly positively influenced by plant leaf nitrogen content and negatively influenced by plant standing biomass and plant specific root length (Fig. S5, Supporting information). Among these variables, plant leaf nitrogen content, specific root length and standing biomass all had relative importance values of $100 \%$ in the lowest-AIC scoring models.

In the statistical models assessing the effects of plant phylum, plant origin and block on fungal richness, results were consistent between fungal guilds except for plant phylum. Neither block nor plant origin had a significant effect on ectomycorrhizal or saprotrophic fungal richness (Fig. S6, Supporting information). In contrast, plant phylum had a significant effect on ectomycorrhizal fungal richness $\left(F_{2,47}=92.29, P<0.004\right)$, but not saprotrophic fungal richness (Fig. 1). Species richness did not differ between the gymnosperm-only and angiosperm-only plots for ectomycorrhizal fungi, but was significantly higher in the plots containing both gymnosperm and angiosperm host trees (Tukey HSD test, $P<0.05$ ). Although the total number of ectomycorrhizal fungal species present in plots with both host types was higher, there were no ectomycorrhizal species unique to those plots (Table 2). Instead, many of the species present in either the gymnosperm-only or the angiosperm-only plots were also present in the mixedhost plots.

Comparing fungal species composition across plots, Bray-Curtis dissimilarities among both the ectomycorrhizal and saprotrophic fungal guilds were significantly correlated with Bray-Curtis as well as Raup-Crick dissimilarities in plant species composition (ectomycorrhizal fungal Bray-Curtis/plant Bray-Curtis Mantel test: $r=0.409, P<0.001$; ectomycorrhizal fungal BrayCurtis/plant Raup-Crick Mantel test: $r=0.423$, $P<0.001$; saprotrophic fungal Bray-Curtis/plant BrayCurtis Mantel test: $r=0.123, P<0.001$; saprotrophic fungal Bray-Curtis/plant Raup-Crick Mantel test: $r=0.13, \quad P<0.001)$. For both ectomycorrhizal and
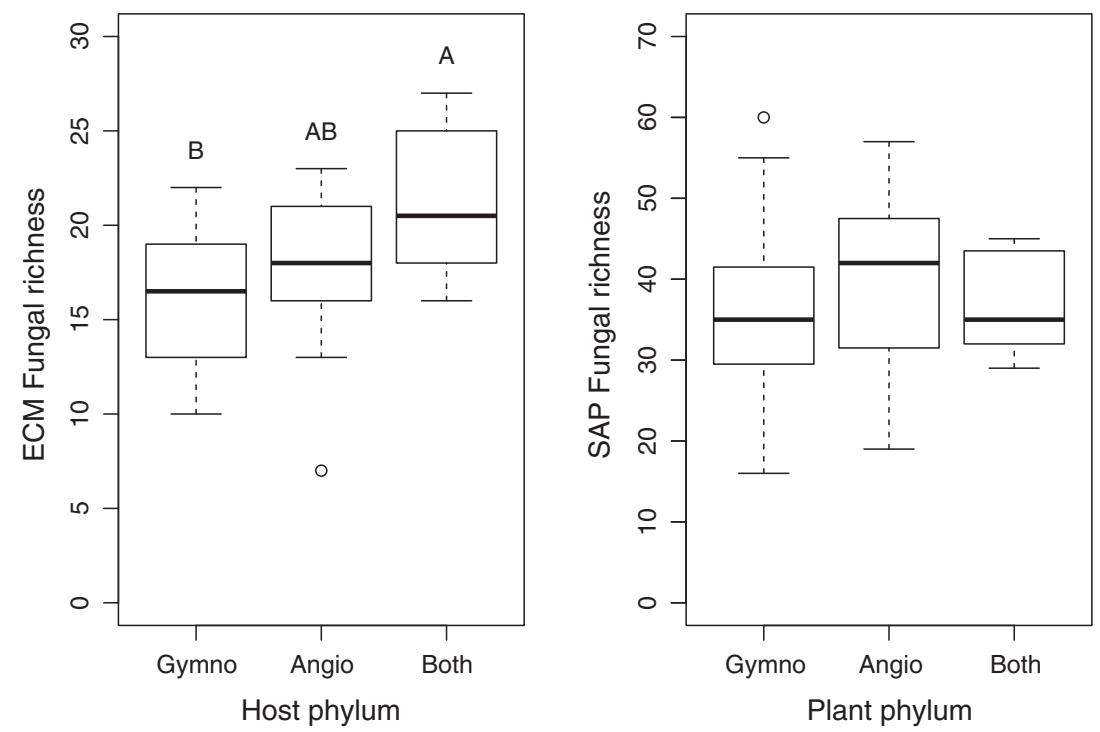

Fig. 1 Ectomycorrhizal (ECM) and saprotrophic (SAP) fungal species richness in plots containing gymnosperm plants only (gymno), angiosperm plants only (angio) or both types of plants (both) in the Cloquet IDENT experiment. Host and plant phylum differ only in the exclusion of the Acer-only plots in the ECM fungal analyses. Boxes surrounding median richness values represent the first and third quartiles, and whiskers show the maximum and minimum values excluding outliers (which are represented by dots). Phylum groups not sharing the same letter are significantly different based on a Tukey HSD test. ANOVA model $r^{2}=0.21$. 
Table 2 Ectomycorrhizal (ECM) fungal species present in 6 angiosperm plots, 6 gymnosperm plots, or mixed-host (i.e. both) plots in the Cloquet IDENT experiment

\begin{tabular}{|c|c|c|}
\hline Plot type & Species & ECM fungal species name best match \\
\hline Gymno only & 3 & Amphinema byssoides (SH229867), Amphinema sp. (SH229877), Suillus cavipes (SH190974) \\
\hline Angio only & 8 & $\begin{array}{l}\text { Clavulina sp. (SH001392), Cortinarius sp. (SH010342), Laccaria sp. (SH205134), Lactarius sp. } \\
\text { (SH003059) Leccinum scabrum (SH197538), Leccinum schistophilum (SH197295), Tomentella } \\
\text { sublilacina (SH004591), Thelephora sp. (SH219948) }\end{array}$ \\
\hline Gymno + Both & 11 & $\begin{array}{l}\text { Amphinema byssoides (SH229867), Amphinema sp. (SH229877), Inocybe lanatodisca (SH215203), } \\
\text { Laccaria laccata (SH205145), Meliniomyces sp. (SH012500), Rhizopogon roseolus (SH001018), } \\
\text { Russula sp. (SH025246), Russula sp. (SH20839), Suillus sp. (SH000892), Suillus grevillei } \\
\text { (SH19095), Tomentella botryoides (SH202494), Tomentella sp. (SH009549) }\end{array}$ \\
\hline Angio + Both & 11 & $\begin{array}{l}\text { Cortinarius alborufescens (SH023149), Cortinarius argyrionus (SH191810), Cortinarius sp. } \\
\text { (SH031729), Cortinarius sp. (SH031772), Entoloma rhodopolium (SH038256), Inocybe sp. } \\
\text { (SH200564), Laccaria sp. (SH010997), Laccaria laccata (SH205145), Meliniomyces sp. } \\
\text { (SH207199), Tomentella sp. (SH219853), Tuber sp. (SH200481) }\end{array}$ \\
\hline Both only & 0 & \\
\hline
\end{tabular}

saprotrophic fungal guilds, fungal species composition was more similar in plots containing more similar plant species, however, the amount of change in ectomycorrhizal fungal species composition per change in plant species composition was notably higher than for saprotrophic fungi (Fig. 2). The two guilds also differed in the response of species composition to both plant origin and plant phylum. Ectomycorrhizal fungal species composition differed significantly by plant phylum (PERMANOVA: $\left.F_{2,50}=4.82, \quad P<0.001, r^{2}=0.153\right)$, with the gymnosperm and angiosperm plots showing the greatest divergence in composition and plots containing both host types being intermediate (Fig. 3). BETADISPER analysis of host phylum effects was not significant (ANOVA: $F_{2,52}=2.452, P=0.096$ ), indicating this was not due to differences in dispersion among groups. Ectomycorrhizal fungal species composition also differed significantly by plant origin (PERMANOVA: $F_{2,50}=1.69$, $\left.P=0.021, r^{2}=0.053\right)$, with plots containing American and European plant species showing the most divergence and plots containing both being intermediate. However, the significant BETADISPER analysis (ANOVA: $\left.F_{2,52}=4.805, P=0.013\right)$ suggested the significant PERMANOVA difference was caused by the greater dispersion in plots with only European or American plant species compared with those with both. In contrast,
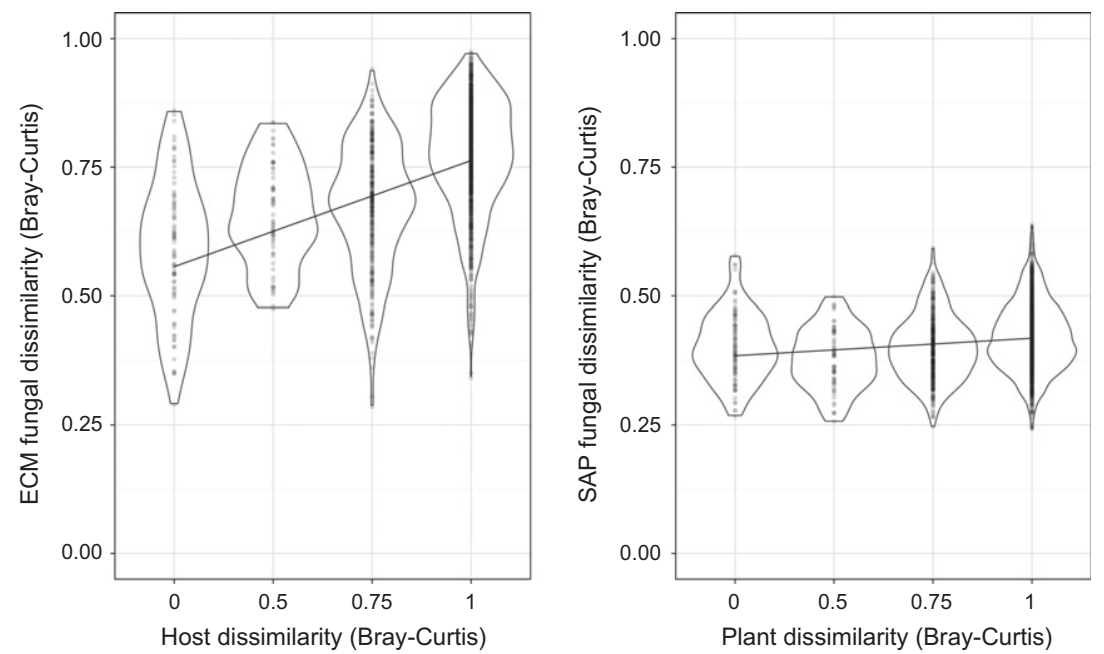

Fig. 2 Bray-Curtis dissimilarities between plant and ectomycorrhizal (ECM) and saprotrophic (SAP) fungal communities in the Cloquet IDENT experiment. Values were plotted on axes ranging between zero and one, where increasing values indicate greater dissimilarity between plot communities. Due to multiple overlapping observations, points were plotted as partially transparent, with darkest reflecting data density. Density curves were plotted to reflect the underlying distribution of fungal community dissimilarity at each level of plant community dissimilarity (a.k.a. violin plots). Linear regression lines were included to indicate trends in the change in fungal dissimilarity with changes in plant community dissimilarity. 

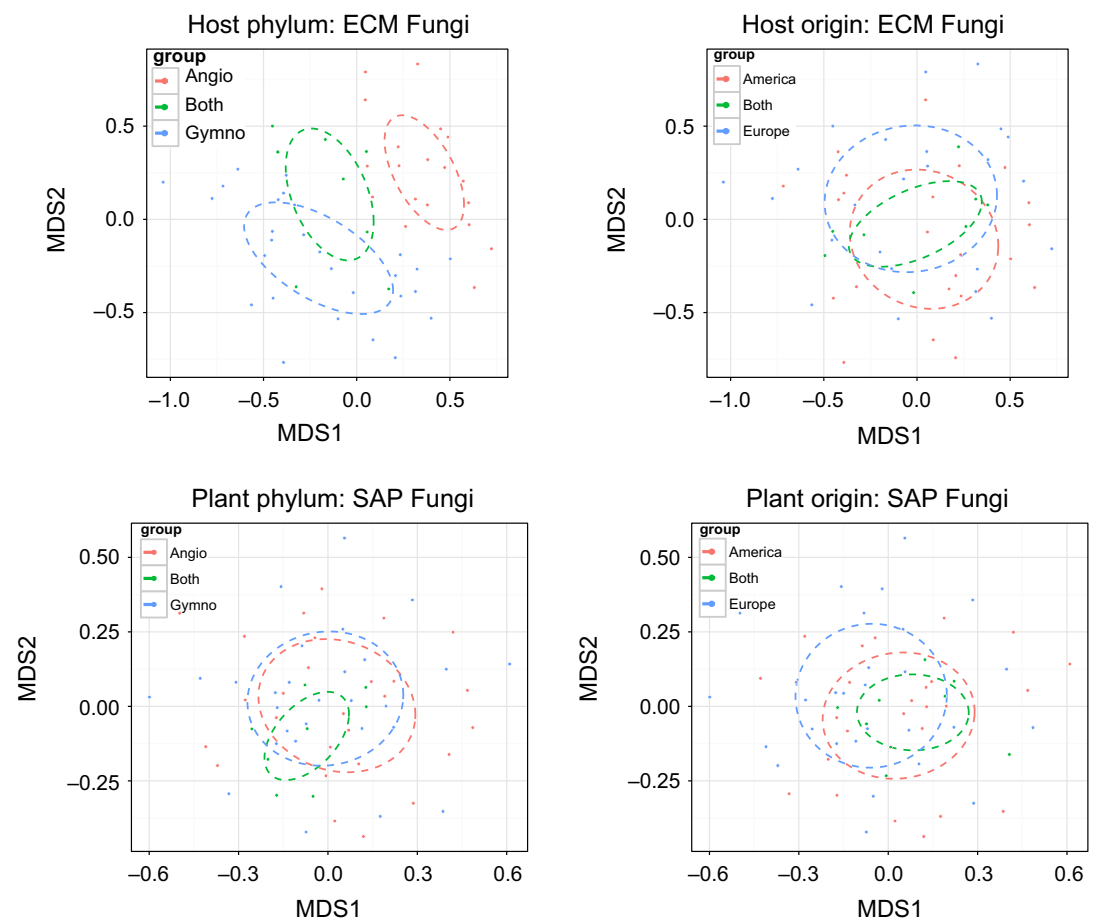

Fig. 3 Nonmetric multidimensional scaling plots of ectomycorrhizal (ECM) and saprotrophic (SAP) fungal species composition at the Cloquet IDENT experiment. Points in ordination space represent individual plots, with colours representing plant phylum (angiosperm, gymnosperm, both) or plant origin (America, Europe, Both). Standard deviation ellipses were projected on the ordination plot to visualize multivariate dispersion in community composition by plant phylum and plant origin. saprotrophic fungal species composition did not respond significantly to either variable (plant phylum PERMANOVA: $F_{2,58}=0.91, P=0.646, r^{2}=0.029$, plant origin PERMANOVA: $F_{2,58}=1.16, P=0.197, r^{2}=0.038$ ).

\section{Discussion}

Our results are the first to experimentally validate that the significant positive relationships between plant phylogenetic diversity and ectomycorrhizal fungal species richness and composition are not confounded by sampling effects (Ishida et al. 2007; Tedersoo et al. 2012, 2013, 2014a; Gao et al. 2013, 2014). The absence of a similar response for soil saprotrophic fungi supports the growing consensus that the nature of the relationship between plant diversity and soil fungal richness depends significantly on fungal guild (Peay et al. 2013; Tedersoo et al. 2014a, 2015). Interestingly, we found no significant effect of increasing plant species richness on fungal species richness for either ectomycorrhizal or saprotrophic fungi. While these results are consistent with the findings of Prober et al. (2014), other studies have found a significantly positive relationship between plant richness and either mycorrhizal or saprotrophic fungal guilds (Peay et al. 2013; Hiiesalu et al. 2014; Pellisier et al. 2014; Tedersoo et al. 2015). While many factors may contribute the difference between our results and those in other studies (e.g. differences in regional species pools, examination of different aged hosts, focusing on only on soil rather than root fungal communities), one that we think is particularly notable is the relatively low richness of plants present in the Cloquet IDENT plots. The maximum plot plant richness at the study site was just six species, which is an order of magnitude lower than all but one of the aforementioned studies. This difference suggests the effect of plant species richness on soil fungal richness may be relatively small and that studies incorporating larger plant species gradients ( $>20$ species) are needed to clearly observe the cumulative nature of this relationship.

In addition to demonstrating a link between plant phylogenetic diversity and ectomycorrhizal fungal richness and composition, our results may also shed light on the mechanism by which plant and ectomycorrhizal fungal richness are connected. It appears the influence of plant phylogenetic diversity on ectomycorrhizal fungal richness is driven by host specificity rather than any chemical or physiological synergy among different host trees. Had the copresence of gymnosperms and angiosperms uniquely facilitated the growth of certain ectomycorrhizal fungal species, we would have expected to see a set of species only present in the mixed-host plots. However, we found no ectomycorrhizal fungal species unique to the six North American and six European plots (which contained both gymnosperm and angiosperm hosts) (Table 2). Instead, we found many of the ectomycorrhizal fungal species present in gymnosperm-only plots but not angiosperm-only plots (and vice versa) were also present in the mixed-host plots. While we 
did not sample roots and therefore cannot be certain of the presence of functional mycorrhizas between specialist species and their known hosts in mixed-host plots, this latter pattern is consistent with a specificity effect, that is only in the presence of particular hosts are certain ectomycorrhizal fungal species also present. Examining the identity of species further confirmed this pattern, with known ectomycorrhizal fungal host specialists such as Suillus spp. and Rhizopogon roseolus on gymnosperm hosts and Leccinum spp. on angiosperm hosts only occurring in plots where particular hosts were present. While the specificity patterns were strong at interphylum level (gymnosperm vs. angiosperm), we found little evidence that intraphylum (within gymnosperm or within angiosperm) specificity was a significant factor driving ectomycorrhizal fungal richness patterns. Specifically, we did not observe a significant increase in ectomycorrhizal fungal species richness in the six gymnosperm or six angiosperm plots, compared with the monoculture plots of each type, respectively (Fig. S7, Supporting information). Although our power to test intraphylum effects was weaker than those between phyla, these results suggest ectomycorrhizal fungal specificity effects may be most consistently apparent at higher (e.g. order and phylum) than lower (e.g. species and genus) plant taxonomic levels (Ishida et al. 2007; Gao et al. 2013; Tedersoo et al. 2013).

Despite being more than twice as species rich as the ectomycorrhizal fungal communities, the saprotrophic fungal communities had less than half the number of sequences in the plots. This suggests that ectomycorrhizal fungi dominated the soil fungal communities in the Cloquet IDENT plots, which is not surprising given the high host density present. The highly guild-dependent results that we observed in fungal species composition parallel the results of Peay et al. (2013), who found that symbiotic fungal community richness responded more strongly to plant community changes than that of saprotrophic fungi. While differential responses between these fungal guilds seem likely given to the varying nature of their carbon sources, we believe the nature of our study site may also have been an important contributing factor. The Cloquet IDENT site that we sampled is a relatively young forest setting (5 years old at the time of sampling) and, although some of the trees were well over $2 \mathrm{~m}$ tall, there has been limited soil organic matter accumulation in the plots (the soil cores were also sieved, so larger coarse organic material that may have harboured more saprotrophic fungi was also removed). In older forest sites in Europe, both Urbanová et al. (2015) and Tedersoo et al. (2015) found that the richness and composition of soil fungal communities was strongly coupled to presence of particular host trees. Their findings suggest that rather than soil saprotrophic fungal communities being unaffected by plant phylogenetic diversity, greater differences in the saprotrophic fungal community are likely to develop with time, as the differential effects of host on litter chemistry and quality accumulate. Along with differences in responses related to plant composition, the saprotrophic fungal communities in our study also had greater overall homogeneity (i.e. consistently lower dissimilarity in all plots) than the ectomycorrhizal fungal communities. Although the reasons for this pattern are not clear, they do not appear to be related to differences in species richness between the two fungal guilds, as Peay et al. (2013) found a similar pattern between mycorrhizal and saprotrophic fungi despite having nearly identical species richness.

The positive correlation between saprotrophic fungal richness and host leaf nitrogen can be interpreted in multiple ways. It is possible that more nitrogen-rich litter may support a greater number of saprotrophic fungal species by decreasing competition for this resource. However, it has been widely shown that soil saprotrophic fungi are more carbon than nitrogen limited (Dighton 2003), which does not support this interpretation. Reich (2012) showed that leaf nitrogen content is strongly correlated with site productivity in temperate and boreal forests, likely due to the nitrogen present in photosynthetic enzymes. Given this pattern, it is also possible that the increase in saprotrophic fungal richness with leaf nitrogen content that we observed may be due to greater carbon inputs, either through greater rhizosphere $\mathrm{C}$ deposition or increased leaf litter (Broeckling et al. 2008). The negative correlation between soil saprotrophic richness and specific root length was surprising, given that leaf nitrogen content and specific root length were themselves positively correlated (Fig. S8, Supporting information). While we did not collect any data on fungal biomass in these plots, it is possible that greater specific root length may have favoured the growth of ectomycorrhizal fungal species by providing additional root tips for colonization. As previous work has shown that ectomycorrhizal fungi can suppress the growth of saprotrophic fungi (Gadgil \& Gadgil 1975; but see Shaw et al. 1995), greater abundances of ectomycorrhizal fungi may be responsible for the negative relationship between specific root length and saprotrophic fungal richness. Clearly, additional studies are needed to better understand the mechanisms driving saprotrophic fungal richness at local spatial scales.

Plant origin did not have strong effects on ectomycorrhizal fungal species richness, but it did significantly affect ectomycorrhizal fungal species composition. While this difference appeared to largely associated 
with less variation in species composition across plots with both American and European hosts compared with those with only American or only European hosts, we believe a close inspection of members of the genera Rhizopogon, Suillus and Leccinum provides additional insight. These genera contain a number of species with known geographic distributions, both in North America and Europe. For example, the species Suillus grevillei is known to occur on Larix hosts on both continents and was present in similar abundance in the L. laricina and L. decidua plots. Two other Suillus species, S. pictus and S. intermedius, are known to occur only in North America and were both only present in plots containing the North American host species, Pinus strobus. In contrast, Rhizopogon roseolus, a species of known European origin, was only present in plots containing the European host species, Pinus sylvestris. The two Leccinum species also showed similar differences, with the European species L. schistophilum being only present on the European Betula pendula, and the other species, L. scabrum, while known from both Europe and North America (den Bakker et al. 2007), being only present on North American Betula species, B. papyifera. Collectively, these plot occurrence patterns suggest that specificity due to host origin may also contribute the observed divergences in ectomycorrhizal fungal species composition across plots. At the same time, because the congeneric species occurred with different hosts, this did not strongly affect plot-level ectomycorrhizal fungal richness (which is consistent with the absence of a significant host origin effect on ectomycorrhizal richness). Although more sampling in studies employing similar designs is needed to determine the strength and consistency of these patterns, our results suggest that host origin along with phylogenetic diversity can have important effects of ectomycorrhizal fungal community structure.

\section{Conclusions}

Taken together, our results provide an important experimental test of the nature of plant and soil fungal community relationships. Our findings that host plant phylogenetic diversity is a significant driver of ectomycorrhizal fungal richness and composition is consistent with other observation-based studies and suggests that the global peak in ectomycorrhizal fungal richness at temperate latitudes may be driven by the parallel increase in host plant phylogenetic diversity (Tedersoo et al. 2012, 2014a,b). As other important variables related to ectomycorrhizal fungal richness (e.g. soil organic matter content and host density) are also high in temperate zones, future experimental tests will be important in further refining our understanding of the global drivers of ectomycorrhizal fungal richness (Kennedy et al. 2012). The different responses of soil saprotrophic fungi suggest that while their community richness and composition are influenced by plant community structure, other ecological factors are more influential on nonsymbiotic fungal communities. Similar differences between guilds have been seen in studies at larger spatial scales (Peay et al. 2013; Tedersoo et al. 2014a; but see Prober et al. 2014), suggesting the patterns we observed in this local-scale study are robust. Fortunately, the pairing of highthroughput sequencing, which captures all fungi present in samples, and the better automation of parsing fungi by ecological guild (Nguyen et al. 2016) will greatly facilitate the parallel study of symbiotic and free-living fungal communities. This will allow researchers to more clearly identify the important ecological similarities and differences among fungal guilds, which will ultimately help in better understanding their effects on ecosystem diversity and functioning.

\section{Acknowledgements}

The authors gratefully thank the many field assistants for the set-up and maintenance of the Cloquet IDENT experiment. Helpful advice about analytical approaches was provided by W. Pearse, J. Grossman and A. David. Constructive comments on previous drafts were kindly provided by W. Harcombe, F. Isbell, J. Schilling, members of the UMN Mycology Club reading group and three anonymous reviewers.

\section{References}

Altschul SF, Gish W, Miller W, Myers EW, Lipman DJ (1990) Basic local alignment search tool. Journal of Molecular Biology, 215, 403-410.

Antoninka A, Reich PB, Johnson NC (2011) Seven years of carbon dioxide enrichment, nitrogen fertilization and plant diversity influence arbuscular mycorrhizal fungi in a grassland ecosystem. New Phytologist, 192, 200-214.

den Bakker HC, Zuccarello GC, Kuyper TW, Noordeloos ME (2007) Phylogeographic patterns in Leccinum sect. Scabra and the status of the arctic-alpine species L. rotundifoliae. Mycological Research, 111, 663-672.

Bardgett RD, Van der Putten WH (2014) Belowground biodiversity and ecosystem functioning. Nature, 515, 505-511.

Broeckling CD, Broz AK, Bergelson J, Manter DK, Vivanco JM (2008) Root exudates regulate soil fungal community composition and diversity. Applied and Environmental Microbiology, 74, 738-744.

Bryant JA, Lamanna C, Morlon $\mathrm{H}$ et al. (2008) Microbes on mountainsides: contrasting elevational patterns of bacterial and plant diversity. PNAS, 105(Suppl), 11505-11511.

Burrows RL, Pfleger FL (2002) Arbuscular mycorrhizal fungi respond to increasing plant diversity. Canadian Journal of Botany, 80, 120-130. 
Caporaso JG, Lauber CL, Walters WA et al. (2011) Global patterns of 16S rRNA diversity at a depth of millions of sequences per sample. PNAS, 108, 4516-4522.

Carlsen T, Aas AB, Lindner D et al. (2012) Don't make a mista (g)ke: Is tag switching an overlooked source of error in amplicon pyrosequencing studies? Fungal Ecology, 5, 747-749.

Chase JM, Kraft NJB, Smith KG et al. (2011) Using null models to disentangle variation in community dissimiliarity from variation in alpha-diversity. Ecosphere, 2, art24.

Chave J, Coomes D, Jansen S et al. (2009) Towards a worldwide wood economics spectrum. Ecology Letters, 12, 351-366.

De Deyn GB, Van der Putten WH (2005) Linking aboveground and belowground diversity. Trends in Ecology and Evolution, 20, 625-633.

Dighton J (ed.) (2003) Fungi in Ecosystem Processes. CRC Press, Boca Raton, Florida.

Eisenhauer N, Milcu A, Sabais ACW et al. (2011) Plant diversity surpasses plant functional groups and plant productivity as driver of soil biota in the long term. PLOS ONE, 6, e16055.

Fierer N, Jackson RB (2006) The diversity and biogeography of soil bacterial communities. PNAS, 103, 626-631.

Gadgil RL, Gadgil PD (1975) Suppression of litter decomposition by mycorrhizal roots of Pinus radiata. New Zealand Journal of Forestry Science, 5, 33-41.

Gao C, Shi N-N, Liu Y-X et al. (2013) Host plant genus-level diversity is the best predictor of ectomycorrhizal fungal diversity in a Chinese subtropical forest. Molecular Ecology, 22, 3403-3414.

Gao C, Shi N-N, Liu Y-X et al. (2014) Host plant richness explain diversity of ectomycorrhizal fungi: response to the comment of Tedersoo et al. (2014). Molecular Ecology, 23, 996-999.

Gilbert GS, Webb CO (2007) Phylogenetic signal in plant pathogen-host range. Proceedings of the National Academy of Sciences, 104, 4979-4983.

Haddad NM, Crutsinger GM, Gross K et al. (2009) Plant species loss decreases arthropod diversity and shifts trophic structure. Ecology Letters, 12, 1029-1039.

Helmus MR, Bland TJ, Williams CK, Ives AR (2007) Phylogenetic measures of biodiversity. The American Naturalist, 169, E68-E83.

Hiiesalu I, Pärtel M, Davison J et al. (2014) Species richness of arbuscular mycorrhizal fungi: associations with grassland plant richness and biomass. New Phytologist, 203, 233-244.

Hood-Nowotny R, Umana NH, Inselbacher E, OswaldLachouani P, Wanek W (2010) Alternative methods for measuring inorganic, organic, and total dissolved nitrogen in soil. Soil Science Society of America Journal, 74, 1018-1027.

Hooper DU, Chapin FS, Ewel JJ et al. (2005) Effects of biodiversity on ecosystem functioning: a consensus of current knowledge. Ecological Monographs, 75, 3-35.

Huston MA (1997) Hidden treatments in ecological experiments: re-evalutating the ecosystem function of biodiverstiy. Oecologia, 110, 449-460.

Ishida TA, Nara K, Hogetsu T (2007) Host effects on ectomycorrhizal fungal communities: insight from eight host species in mixed conifer-broadleaf forests. New Phytologist, 174, 430-440.

Kennedy PG, Matheny PB, Ryberg KM et al. (2012) Scaling up: examining the macroecology of ectomycorrhizal fungi. Molecular Ecology, 21, 4151-4154.
Kernaghan G, Widden P, Bergeron Y, Légaré S, Paré D (2003) Biotic and abiotic factors affecting ectomycorrhizal diversity in boreal mixed-woods. Oikos, 102, 497-504.

Kõljalg U, Nilsson RH, Abarenkov K et al. (2013) Towards a unified paradigm for sequence-based identification of fungi. Molecular Ecology, 22, 5271-5277.

Landis FC, Gargas A, Givnish TJ (2004) Relationships among arbuscular mycorrhizal fungi, vascular plants and environmental conditions in oak savannas. New Phytologist, 164, 493504.

Lauber CL, Hamady M, Knight R, Fierer N (2009) Pyrosequencing-based assessment of soil $\mathrm{pH}$ as a predictor of soil bacterial community structure at the continental scale. Applied and Environmental Microbiology, 75, 5111-5120.

LeBlanc N, Kinkel LL, Kistler HC (2015) Soil fungal communities respond to grassland plant community richness and soil edaphics. Microbial Ecology, 70, 188-195.

Lindahl BD, Nilsson RH, Tedersoo L et al. (2013) Fungal community analysis by high-throughput sequencing of amplified markers - a user's guide. New Phytologist, 199, 288-299.

McMurdie PJ, Holmes S (2014) Waste not, want not: why rarefying microbiome data is inadmissible. PLoS Computational Biology, 10, e1003531.

Molina R, Massicotte HB, Trappe JM (1992) Specificity phenomena in mycorrhizal symbioses: community-ecological consequences and practical implications. In: Mycorrhizal Functioning: An Integrative Plant-Fungal Process (ed. Allen MF), pp. 357-423. Chapman \& Hall, New York.

Nara K, Hogetsu T (2004) Ectomycorrhizal fungi on established shrubs facilitate subsequent seedling establishment of successional plant species. Ecology, 85, 1700-1707.

Nguyen NH, Smith DP, Peay KG, Kennedy PG (2015) Parsing ecological signal from noise in next generation amplicon sequencing. New Phytologist, 205, 1389-1993.

Nguyen NH, Song Z, Bates ST et al. (2016) FUNGuild: an open annotation tool for parsing fungal community datasets by ecological guild. Fungal Ecology, 20, 241-248.

Pearse W, Cavender-Bares J, Helmus MR (2014) Metrics and models of community phylogenetics. In: Modern Phylogenetic Comparative Methods and their Application in Evolutionary Biology: Concepts and Practice (ed. Garamszegi L), pp. 451-464. Springer, Berlin.

Peay KG, Kennedy PG, Bruns TD (2008) Fungal community ecology: a hybrid beast with a molecular master. BioScience, 58, 799-810.

Peay KG, Baraloto C, Fine PVA (2013) Strong coupling of plant and fungal community structure across western Amazonian rainforests. ISME Journal, 7, 1852-1861.

Pellisier L, Niculita-Hirzel H, Dubuis A et al. (2014) Soil fungal communities of grasslands are spatially structured at a regional scale in the Alps. Molecular Ecology, 23, 42744290.

Pregitzer KS, DeForest JL, Burton AJ et al. (2002) Fine root architecture of nine North American trees. Ecological Monographs, 72, 293-309.

Prober SM, Leff JW, Bates ST et al. (2014) Plant diversity predicts beta but not alpha diversity of soil microbes across grasslands worldwide. Ecology Letters, 18, 85-95.

R Core Team (2014) R: A Language and Environment for Statistical Computing. R Foundation for Statistical Computing, Vienna, Austria. Available from http://www.R-project.org/. 
Reich PB (2012) Key canopy traits drive forest productivity. Proceedings of the Royal Society B: Biological Sciences, 279, 2128-2134

Rottstock T, Joshi J, Kummer V, Fischer M (2014) Higher plant diversity promotes higher diversity of fungal pathogens, while it decreases pathogen infection per plant. Ecology, 95, 1907-1917.

Schloss PD, Westcott SL, Ryabin T et al. (2009) Introducing mothur: open-source, platform-independent, communitysupported software for describing and comparing microbial communities. Applied and Environmental Microbiology, 75, 7537-7541.

Shaw TM, Dighton J, Sanders FE (1995) Interactions between ectomycorrhizal and saprotrophic fungi on agar and in association with seedlings of lodgepole pine (Pinus contorta). Mycological Research, 99, 159-165.

Smith DP, Peay KG (2014) Sequence depth, not PCR replication, improves ecological inference from next generation DNA sequencing. PLoS ONE, 9, e90234.

Smith LM, Reynolds HL (2015) Plant-soil feedback shifts from negative to positive with decreasing light in forest understory species. Ecology, 96, 2523-2532.

Soil Survey Staff (2004) Soil survey laboratory methods manual. In: Soil Survey Investigations Report No. 42, Version 4 (ed. Burt R), pp. 204-205. U.S. Department of Agriculture, Natural Resources Conservation Service.

Song Z, Schlatter D, Kennedy P et al. (2015) Effort vs. reward: preparing samples for fungal community characterization in high-throughput sequencing surveys of soils. PLOS ONE, 10, e0127234

Tedersoo L, Bahram M, Toots M et al. (2012) Towards global patterns in the diversity and community structure of ectomycorrhizal fungi. Molecular Ecology, 21, 4160-4170.

Tedersoo L, Mett M, Ishida TA, Bahram M (2013) Phylogenetic relationships among host plants explain differences in fungal species richness and community composition in ectomycorrhizal symbiosis. New Phytologist, 199, 822-831.

Tedersoo L, Bahram M, Põlme S et al. (2014a) Global diversity and geography of soil fungi. Science, 346, 1-10.

Tedersoo L, Bahram M, Dickie IA (2014b) Does host plant richness explain diversity of ectomycorrhizal fungi? Re-evaluation of Gao et al. (2013) data sets reveals sampling effects Molecular Ecology, 23, 992-995.

Tedersoo L, Bahram M, Cajthaml T et al. (2015) Tree diversity and species identity effects on soil fungi, protists and animals are context dependent. The ISME Journal, 00, 1-17.

Thomas SC, Winner WE (2000) Leaf area index of an oldgrowth Douglas-fir forest estimated from direct structural measurements in the canopy. Canadian Journal of Forest Research, 30, 1922-1930.

Tobner CM, Paquette A, Reich PB, Gravel D, Messier C (2014) Advancing biodiversity-ecosystem functioning science using high-density tree-based experiments over functional diversity gradients. Oecologia, 174, 609-621.

Urbanová M, Šnajdr J, Baldrian P (2015) Composition of fungal and bacterial communities in forest litter and soil is largely determined by dominant trees. Soil Biology and Biochemistry, 84, 53-64.

Van der Heijden MG, Klironomos JN, Ursic M et al. (1998) Mycorrhizal fungal diversity determines plant biodiversity, ecosystem variability and productivity. Nature, 396, 69-72.
Van der Heijden MGA, Bardgett RD, Van Straalen NM (2008) The unseen majority: soil microbes as drivers of plant diversity and productivity in terrestrial ecosystems. Ecology Letters, 11, 296-310.

Van der Linde S, Haller S (2013) Obtaining a spore free fungal community composition. Fungal Ecology, 6, 522-526.

Van der Putten WH, Van Dijk C, Peters BAM (1993) Plant-specific soil-borne diseases contribute to succession in foredune vegetation. Nature, 362, 53-56.

Vogelsang KM, Reynolds HL, Bever JD (2006) Mycorrhizal fungal identity and richness determine the diversity and productivity of a tallgrass prairie system. New Phytologist, 172, 554-562.

Wagg C, Bender SF, Widmer F, Van der Heijden MGA (2014) Soil biodiversity and soil community composition determine ecosystem multifunctionality. PNAS, 111, 5266-5270.

Waldrop MP, Zak DR, Blackwood CB, Curtis CD, Tilman D (2006) Resource availability controls fungal diversity across a plant diversity gradient. Ecology Letters, 9, 1127-1135.

Wardle DA (2006) The influence of biotic interactions on soil biodiversity. Ecology Letters, 9, 870-886.

Whittingham MJ, Stephens PA, Bradbury RB, Freckleton RP (2006) Why do we still use stepwise modelling in ecology and behaviour? Journal of Animal Ecology, 75, 11821189.

N.H.N. collected the fungal data and led the initial data analyses. L.J.W. collected the above- and belowground plant data. J.B.V. generated the R simulation code and led the final community composition analyses. A.S. collected the plot physical measures. J.C.-B. assisted with field sampling and provided guidance on the plant phylogenetic diversity analyses. C.M., A.P., D.G. and P.B.R. designed the IDENT experiment and P.B.R. established it at Cloquet. P.G.K. conceived the study, conducted the final statistical analyses, and drafted the manuscript. All authors provided detailed feedback on manuscript drafts.

\section{Data accessibility}

All DNA sequence files: MG-RAST Project \#: 13302. Sample x OTU Matrices: Tables S1 and S2 (Supporting information).

\section{Supporting information}

Additional supporting information may be found in the online version of this article.

Fig S1 Cloquet IDENT study design.

Fig. S2 Effects of varying sequence cut-offs on plot-level fungal species occurrence in the ectomycorrhizal (ECM) monoculture plots of the Cloquet IDENT experiment. 
Fig. S3 Species accumulation curves showing sample saturation, variation in sequence abundance and depth of sequencing of ectomycorrhizal (ECM) and saprotrophic (SAP) fungal datasets.

Fig. S4 Bivariate plots of ectomycorrhizal (ECM) fungal richness and the nine predictor variables in the 'MUMIN' multivariate analyses.

Fig. S5 Bivariate plots of saprotrophic (SAP) fungal richness and the nine predictor variables in the 'MUMIN' multivariate analyses.

Fig. S6 Ectomycorrhizal (ECM) and saprotrophic (SAP) fungal species richness by block (A,B,C,D) and plant origin (America, Europe, Both) in the Cloquet IDENT experiment.

Fig. S7 Ectomycorrhizal (ECM) fungal species richness in the angiosperm monoculture plots vs. the 6 angiosperm plots or the gymnosperm monoculture plots vs. the 6 gymnosperm plots in the Cloquet IDENT experiment.

Fig. S8 Pair-wise correlation matrix of the continuous biotic and abiotic variables used to predict ECM fungal species richness in the Cloquet IDENT experiment.

Table S1 Final raw community OTU $\times$ sample matrix for ectomycorrhizal fungi.

Table S2 Final raw community OTU $\times$ sample matrix for saprotrophic fungi.
Table S3 Individual model scores of all 512 models generated using the MUMIN package when examining the effect of 9 continuous variables on ectomycorrhizal fungal richness.

Table S4 Individual model scores of all 512 models generated using the MUMIN package when examining the effect of 9 continuous variables on saprotrophic fungal richness. Models are sorted by AICc score.

Table S5 Results of ectomycorrhizal fungal richness multimodel averaging comparing two subsets of models: delta $<4$ from lowest AICc score or $95 \%$ confidence interval of AICc score.

Table S6 Results of saprotrophic fungal richness multimodel averaging comparing two subsets of models: delta $<4$ from lowest AICc score or $95 \%$ confidence interval of AICc score.

Table S7 Model of ectomycorrhizal fungal richness with lowest AICc score in the full multimodel analysis using the 'dredge' function in MUMIN.

Table S8 Model of saprotrophic fungal richness with lowest AICc score in the full multimodel analysis using the 'dredge' function in MUMIN. 